\title{
Simple and objective method for routine detection of the macular pigment xanthophyll
}

\author{
Dietrich Schweitzer \\ Susanne Jentsch \\ Jens Dawczynski \\ Martin Hammer \\ Augenklinik der Friedrich-Schiller-Universität Jena \\ Experimentelle Ophthalmologie \\ Bachstrasse 18 \\ 07743 Jena, Germany
}

\author{
Ute E.K. Wolf-Schnurrbusch \\ Sebastian Wolf \\ University of Bern \\ University of eye clinic \\ Inselspital Klinik für Augenheilkunde \\ Bern, Switzerland 3010
}

\begin{abstract}
A new simple method for two-dimensional determination of optical density of macular pigment xanthophyll (ODx) in clinical routine is based on a single blue-reflection fundus image. Individual different vignetting is corrected by a shading function. For its construction, nodes are automatically found in structureless image regions. The influence of stray light in elderly crystalline lenses is compensated by a correction function that depends on age. The reproducibility of parameters in a one-wavelength reflection method determined for three subjects $(47,61$, and 78 years old) was: maxODx $=6.3 \%$, meanODx $=4.6 \%$, volume $=6 \%$, and area $=6 \%$ already before stray-light correction. ODx was comparable in pseudophakic and in an eye with a crystalline lens of the same 11 subjects after stray-light correction. Significant correlation in ODx was found between the one-wavelength reflection method and the two-wavelength autofluorescence method for pseudophakic and cataract eyes of 19 patients suffering from dry age-related macular degeneration (AMD) $\left(R^{2}=0.855\right)$. In pseudophakic eyes, maxODx was significantly lower for dry AMD $(n=45)$ $(\mathrm{ODx}=0.491 \pm 0.102 \mathrm{ODU})$ than in eyes with healthy fundus $(n=22)$ (ODx $=0.615 \pm 0.103$ ODU $) \quad(p=0.000033)$. Also in eyes with crystalline lens, maxODx was lower in AMD $(n=125)$ (ODx $=0.610 \pm 0.093$ ODU) than in healthy subjects $(n=45)$ (ODx $=0.674 \pm 0.098$ ODU) $(p=0.00019)$. No dependence on age was found in the pseudophakic eyes both of healthy subjects and AMD patients. $\odot 2010$ Society of Photo-Optical Instrumentation Engineers. [DOI: 10.1117/1.3526358]
\end{abstract}

Keywords: macular pigment xanthophyll; objective detection; stray light; blue light; reflection; age-related macular degeneration; pseudophakic eye.

Paper 10076SSRR received Feb. 15, 2010; revised manuscript received Oct. 25, 2010; accepted for publication Nov. 1, 2010; published online Dec. 17, 2010.

\section{Introduction}

Age-related macular degeneration (AMD) is the leading source for legal blindness in the elderly population in the western world. ${ }^{1}$ Despite intensive research, its molecular pathophysiology remains inadequately understood. ${ }^{2}$ It is commonly assumed that the accumulation of lipofuscin in the retinal pigment epithelium (RPE) and the formation of drusen at Bruch's membrane play an important role in the development of AMD.

The component $\mathrm{A} 2 \mathrm{E}$ of lipofuscin is formed in the visual cycle by reaction of two all-trans-retinal molecules and one ethanolamine molecule. ${ }^{3}$ The accumulation of lipofuscin and the formation of drusen can be partly assumed as result of a reduced lysosomal activity in cells of retinal pigment epithelium ${ }^{4,5}$ and of a decline in permeability of Bruch's membrane. ${ }^{6}$ Lipofuscin generates reactive oxygen species when exposed to visible light irradiation $^{7,8}$ and sensitizes RPE to blue light. ${ }^{9}$

The lifelong formation and activity of free radicals might be one reason for AMD. On the other hand, a high concentration of the macular pigment xanthophyll might reduce the risk of AMD,

Address all correspondence to: Dietrich Schweitzer, Augenklinik der FriedrichSchiller-Universität Jena, Experimentelle Ophthalmologie, Bachstrasse 18, 07743 Jena, Germany; Tel: 49-3641-933027; E-mail: Dietrich.schweitzer@med. uni-jena.de because it absorbs energy-rich blue radiation, which contributes to the formation of free radicals. Furthermore, xanthophyll acts as scavenger for free radicals. ${ }^{10-12}$ The chromatic aberration of the eye is also reduced by the absorption of blue light. ${ }^{13}$ Xanthophyll quenches singlet oxygen and inhibits the peroxydation of membrane phospholipids. ${ }^{14}$ In animal studies, an inverse relation was found between the concentrations of xanthophyll and of lipofuscin. ${ }^{2}$

The macular pigment xanthophyll consists mainly of lutein and zeaxanthin. ${ }^{15}$ Mesozeaxanthin is formed inside the retina. ${ }^{16}$ According to microphotometric studies, xanthophyll is located in receptor axons (Henle's layer) and in the inner plexiform layer. ${ }^{17}$ In the fovea, zeaxanthin has a concentration twice that of lutein but, in the periphery, the reverse is the case. ${ }^{16}$

Assuming the formation of free radicals is one cause of the development of AMD, a high concentration of xanthophyll in the retina might offer protection against light-induced oxidative damage and a low concentration might indicate a risk of AMD.

In order to confirm a loss of protection, a reduced optical density of macular pigment xanthophyll (ODx) should be detectable in vivo in AMD patients. In contrast, the intake of a lutein-rich diet or a supplement with lutein and zeaxanthin should decrease the incidence of AMD.

1083-3668/2010/15(6)/061714/10/\$25.00 @ 2010 SPIE 
This effect can be demonstrated by double-blind prospective studies carried out over several years. Contradictory results are to be found in the literature. A comparison of studies was made by a meta analysis. ${ }^{18}$ An inverse ratio between the intake of lutein and zeaxanthin and the incidence of AMD was reported. ${ }^{19-21}$ In particular, it was found that visual acuity also improved with this supplement. No reduction in the risk of early AMD was found after supplementing lutein and zeaxanthin. ${ }^{22-26}$

One reason for these discrepant results might be the different study protocols, determining the intake of lutein and zeaxanthin using questionnaires, and missed measurements of changes in the optical density of the macular pigment.

An increased optical density of xanthophyll after supplementation with lutein and zeaxanthin has been measured with different methods. In some pioneering studies, the maximal optical density of xanthophyll was determined by subjective heterochrome flicker photometry. ${ }^{27-29}$ In order to achieve an objective method of determining the two-dimensional distribution of xanthophyll, the two-wavelength reflectometry method was realized in laser-scanning ophthalmoscopy. ${ }^{30}$ The optical density of macular pigment was also determined by approximation of the macular reflection spectra by a mathematical model representing the absorbance spectra of xanthophyll and other absorbers abundant at the fundus. ${ }^{31-33}$ Particularly interesting results were achieved in directional spectral reflection measurements from the fovea, where only the crystalline lens and xanthophyll influence the reflection spectrum. ${ }^{34}$ By developing an imaging fundus spectrometer, locally resolved reflectance spectra were measured along a line crossing the fovea. ${ }^{35}$ Xanthophyll has also been determined by approximation of a foveal reflectance spectrum with a model function. As foveal and parafoveal reflectance spectra were determined simultaneously by imaging spectrometry, the influence of lens transmission was eliminated by calculating the logarithmic difference of spectra measured at both fundus sites. The resulting difference spectrum is mainly generated by the extinction of xanthophyll with, in addition, differences in melanin and retinal blood optical densities between the fovea and the para-fovea.

In an elaborate work, the two-wavelength autofluorescence method was developed. ${ }^{36}$ This objective method is realized in some devices, based on the HRA (Heidelberg Retina Angiograph)-laserscanner ophthalmoscope (Heidelberg Engineering, Heidelberg, Germany). Applying this method, no fixation is required and measurements of the optical density of macular pigment are not influenced by stray light in the crystalline lens. Another method was published for measuring the concentration of both carotenoids in the fovea using the Raman activity of lutein and zeaxanthin. ${ }^{37}$ A comparison of principles and equations for calculation of the optical density of macular pigment has been presented, in detail, in the literature. $^{38}$

All these objective methods have specific disadvantages: stray light and absorption of light in the lens, unequal fundus illumination at the two wavelengths used, and unequal relation of fluorescence at two excitation wavelengths. The main obstacle for routine application in clinical practice, however, is the use of techniques requiring highly sophisticated procedures. For this reason, there has been an unfulfilled need for a commonly available technique for measuring the optical density of xanthophyll. ${ }^{39}$
In this paper, a simple method is presented that uses the reflection at only one wavelength, in conjunction with the conventional technique of fundus cameras or laser-scanning ophthalmoscopes. The optical density of macular pigment is calculated by image processing. In elderly subjects, stray light originating from the crystalline lens superimposes the fundus reflection and may interfere with the measurement of the xanthophyll optical density. This influence must be compensated for. Thus, the following steps were performed for the compensation of the stray-light effect, for the comparison to the two-wavelength autofluorescence method, and for comparing the ODx in agematched healthy subjects and patients, suffering from dry AMD. Special attention was taken on measurements in pseudophakic eyes when no stray light originates from the lens, as follows:

1 Measuring a normalized stray-light equivalent as function of age in 232 subjects (mean 69.8, range 45-88 years, group A).

2 Determining the relation between stray-light equivalent and optical density of xanthophyll (no correction for stray light) in 45 subjects (mean 70.7, range 47-86 years, group B, subgroup of A).

3 Calculating a correction function for stray light.

4 Determining the optical density of xanthophyll in $22 \mathrm{IOL}$ (artificial intraocular lens) wearing subjects in one eye (mean 73.7, range 57-85 years, group C), and comparison to 11 eyes with crystalline lens (subgroup of $\mathrm{C}$ ) in the other eye.

5 Comparing the optical density of xanthophyll, determined by the one-wavelength reflection method and twowavelength autofluorescence method in 19 dry AMD patients (mean 74.9 , range $60-87$ years, group D) with pseudophakic eyes or eyes with crystalline lens.

6 Comparing the optical density of xanthophyll in pseudophakic eyes with healthy fundus (group C) and 45 pseudophakic eyes of patients suffering from dry AMD (mean 76.2, range 57-93 years, group E).

7 Comparing the optical density of xanthophyll in eyes with a crystalline lens in 45 healthy subjects (group B) and 125 patients suffering from dry AMD (mean 67, range 50-83 years, group F).

The research in humans followed the tenets of the Declaration of Helsinki. Informed consent was obtained from the subjects after explanation of the measurement.

\section{Method}

\subsection{Principle of the One-Wavelength Method}

The one-wavelength reflection method uses both the local and spectral selectivity of xanthophyll. Local selectivity means that xanthophyll is detectable only in a certain foveal region. Spectral selectivity means that xanthophyll absorbs blue light at wavelengths of $<530 \mathrm{~nm}$, maximally at $460 \mathrm{~nm} .{ }^{40} \mathrm{In}$ addition, this method assumes continuous local alterations only in fundus reflectance. The fundus is illuminated by blue light of one wavelength near the absorption maximum of xanthophyll. Under this illumination, simplistically, the fundus can be considered to be a 
uniform reflecting surface to which increased absorption in the fovea is added.

Retinal vessels appear with increased absorption too and exudates exhibit an increased reflection. The uniform reflection of the paramacular retina is overlaid by shading associated with vignettation of the optics. In addition, nonuniform fundus illumination is caused by errors in adjustment of the ophthalmoscope to the patient's eye, which varies from measurement to measurement. The assumption of the same shading in all fundus images is not valid in the clinical routine. ${ }^{41}$

One common way for shading correction is low-pass filtering. The common drawback of low-pass filtering and similar operations for shading correction consists of the contribution of pixels representing objects (vessels, exudates, drusen or macular pigment itself). In fact, these pixels are not representative for the analysis of irregularities in illumination. Consequently, only such areas should be used for shading correction that do not contain any structure.

The shading function in the interesting central part of a fundus image can be approximated by a three-dimensional parabolic function. For construction of such a paraboloid, nodes must be determined at several fundus sites. Image regions having no internal structure are well suited to be nodes. Such structureless regions were determined by calculating the coefficient of variation in fields of $n \times n$ pixels. ${ }^{42}$ For fields covering a vessel, small exudates, or a druse, the standard deviation of the reflection of all pixels in the field is high and the coefficient of variation rises above a threshold. Such fields were not used as a node.

The shading function was calculated in an annular field centered on the fovea. The inner diameter of this annulus was $\sim 1.2$ times the diameter of the optic disk (PD) and the outer diameter was $\sim 2.4$ PD. The shading function was assumed as the virtual background in the circular foveolar field, where the optical density of xanthophyll was determined.

\subsection{Automatic Finding of Nodes for the Shading Function}

For the purpose of illustration, the determination of nodes for the calculation of the shading function is shown in Fig. 1. In this case, the coefficient of variation was determined in fields of $11 \times 11$ pixels. After calculating the coefficients of variation, all pixels up to the 0.75 quartile were included as nodes for construction of the shading function. To apply the one wavelength reflection method for the determination of ODx in fields of different sizes, the diameter of the optic disk can be used as a reference.

\subsection{Measurement of Xanthophyll Optical Density}

Assuming that the illuminating blue light penetrates the macular pigment twice, the double density (2ODx) of xanthophyll is the logarithm of the shading function divided by the reflectance in the original fundus image. According to Equation (1), for each pixel in the image,

$$
\text { 2ODx }=k \log \frac{\text { shading function }}{\text { measured reflectance }} .
$$

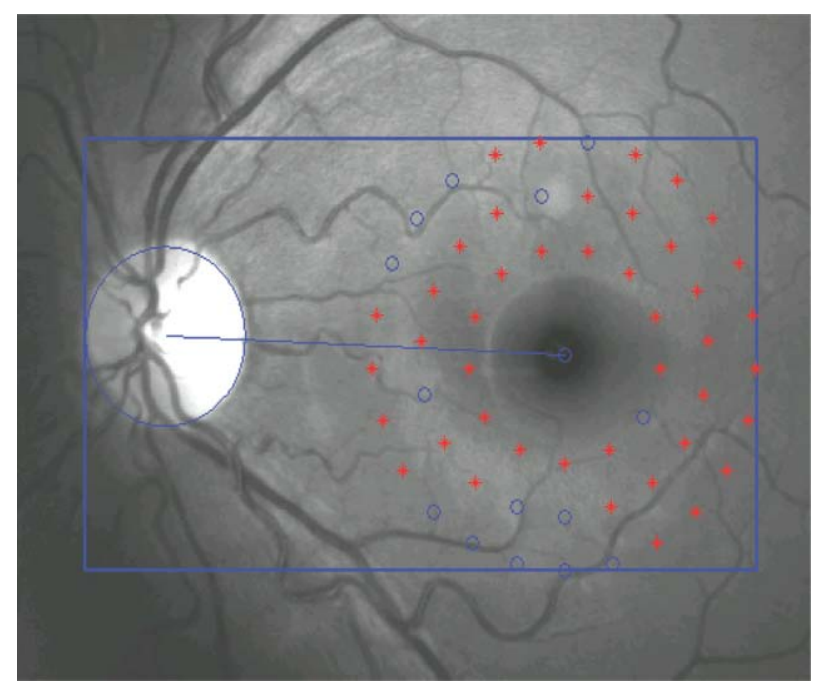

Fig. 1 Principle for determination of nodes for shading function (stars - used, rings - excluded).

The constant $k$ is the ratio of the extinction of xanthophyll in the maximum at $460 \mathrm{~nm}$ and the extinction of xanthophyll at the wavelength of the blue light used. In preliminary studies, values of ODx were found comparable to the results obtained with other methods, if the ratio in Eq. (1) is assumed to be a single optical density. For this reason, all further calculations are considered as single optical densities.

For the calculation of the optical density of xanthophyll, the gray values were recorded by an eight-bit black and white camera. Optical density was determined for each pixel in the circular foveal area. Thus, a quasi three-dimensional distribution of xanthophyll was presented in the macula. In this distribution, four parameters were separated: maximal optical density (max ODx), mean optical density over all pixels (meanODx), the sum of density at all pixels, referred to as "volume," and the area in which the ODx exceeded a threshold. Optical density is measured in optical density units (ODUs), the volume in ODU $\cdot$ square degrees, and the area in square degrees.

In principle, such blue-light fundus images can be taken by any conventional fundus camera in the modus for fluorescence angiography, but without sodium fluorescein injection. Only the cutoff filter must be removed for obtaining blue-light reflection images. All further calculations were carried out using computer software. The individual illumination at the fundus, in particular, was determined by the shading function. This depends on the adjustment of the fundus camera in relation to the eye under investigation. The calculation of the optical density of xanthophyll by the ratio of the shading function and detected foveal reflectance acts as an internal normalization. The individual optical density of macula pigment and its change after supplementation with lutein and zeaxanthin can thus be determined.

\subsection{Influence of Stray Light}

The one-wavelength method has the same problem due to stray light in aging lenses as all reflection methods: the part of the incident light scattered toward the detection system or to the fundus is an additive term $S$, both in the numerator and in the denominator of Eq. (1). Taking scattering $S$ into account, 


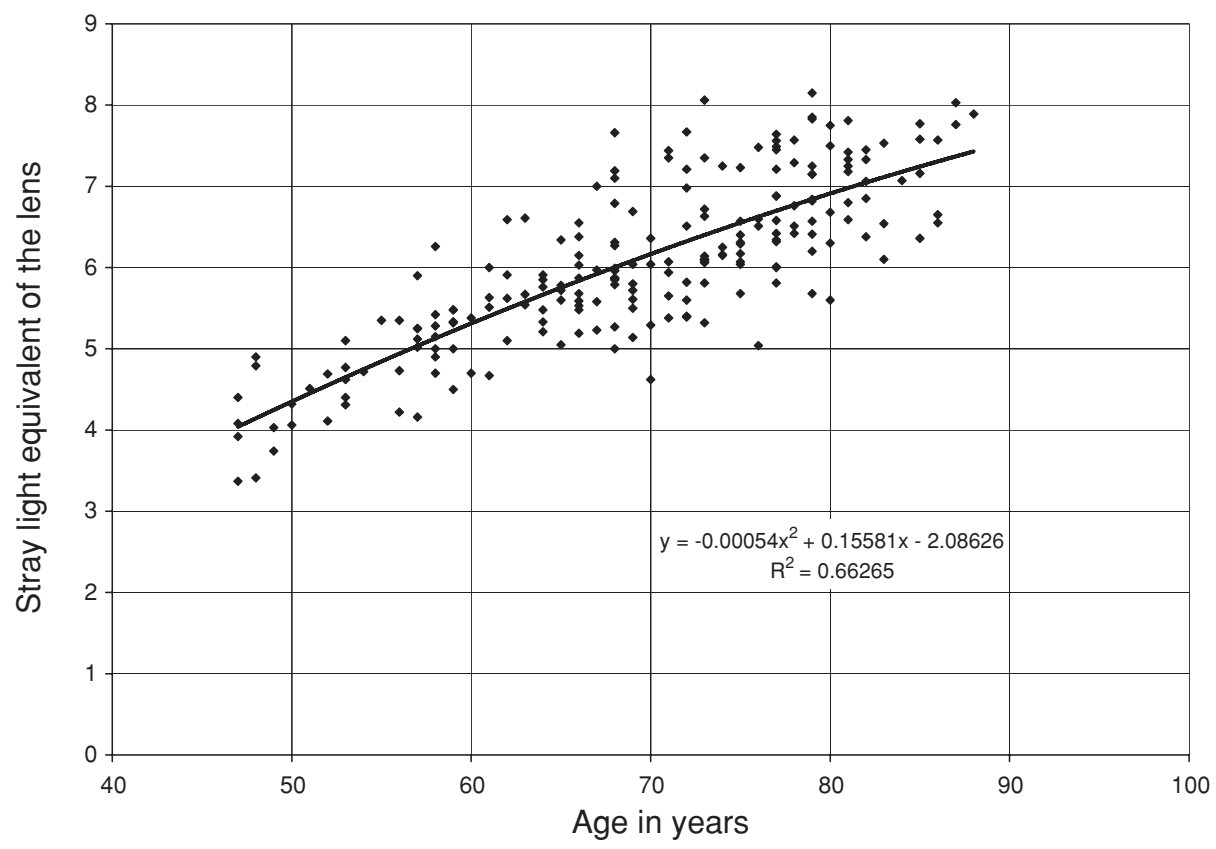

Fig. 2 Normalized stray light equivalent in the lens as a function of age.

Eq. (2) can be derived as follows:

$$
\text { ODx }=k \log \frac{\text { shading function }+S}{\text { measured reflectance }+S} .
$$

According to Eq. (2), scattered light influences xanthophyll measurements as follows. If $S \gg$ shading function and measured reflectance, the limiting value of the ratio in (2) becomes 1 . Thus, a decrease in the calculated ODx can be explained in a progressive cataract because the logarithm of $1=0$.

The influence of stray light was compensated in two steps. First, a normalized stray-light equivalent was determined from 232 patients in the age range between 45 and 88 years (group A), applying the Scheimpflug camera EAS 1000 Nidek (Oculus $\mathrm{GmbH}$, Wetzlar, Germany).All eyes were dilated using one drop of tropicamid (Mydriaticum Stulln ${ }^{\circledR}$, Fa. Pharma Stulln). Second, in 45 subjects (group B, subgroup of A), the decrease in ODx was investigated in relation to the stray-light equivalent in the lens. From both relationships, an age-dependent term was

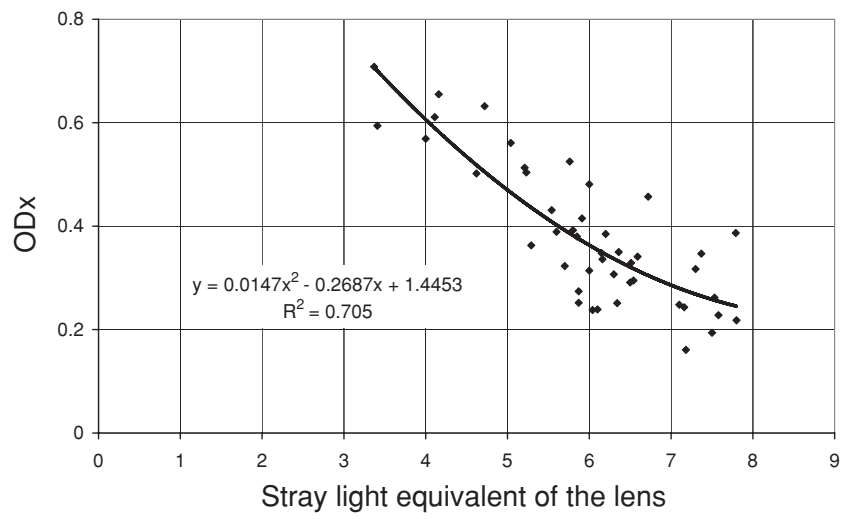

Fig. 3 Decrease in apparent optical density as a function of increasing normalized stray light equivalent. calculated and added to the measured optical density of xanthophyll.

The optical density of xanthophyll was determined in 22 IOLwearing eyes (group C). No stray-light correction was applied in these measurements. The effect of stray-light compensation was tested for 10 subjects with a crystalline lens in one eye and IOL in the other one (subgroup of $\mathrm{C}$ ).

\subsection{Reproducibility}

The reproducibility of mean ODx, maxODx, volume, and area were tested on three subjects, age 47,61 , and 78 years. Ten images were taken from each subject when the fundus camera was completely new and adjusted to the eye. The reproducibility was determined by the coefficient of variation, calculated as the ratio of the standard deviation and the mean values of these ten measurements. The reproducibility was calculated before and after correction for stray light in the lens.

\subsection{Comparison to the Two-Wavelength Autofluorescence Method}

The one-wavelength reflection method is a simplified method for measuring ODx. For its application in routine clinical practice, the correlation between the calculated ODx values and the results obtained by the two-wavelength autofluorescence method was tested. The two-wavelength autofluorescence method is frequently used in recent objective measurement of ODx. ${ }^{43-45}$ For this reason, comparative measurements were made with both systems at the University Eye Hospital Inselspital in Bern, Switzerland.

Xanthophyll measurements by the one-wavelength method were made with a VISUCAM NM/FA ${ }^{\circledR}$ fundus camera (Carl Zeiss Meditec AG, Jena, Germany). The fundus was illuminated in the 30-deg field with flash intensity set to 12 . In addition to the 


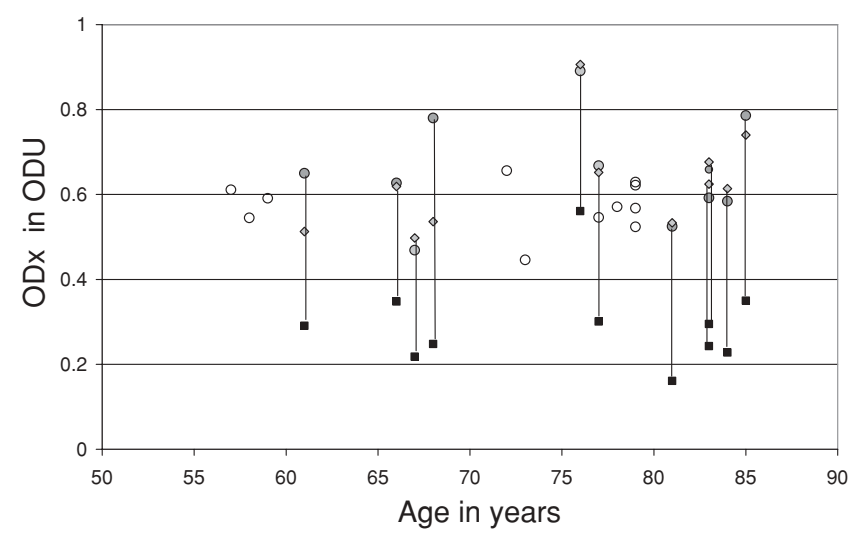

Fig. 4 Comparison of ODx in pseudophakic eyes and in eyes with a crystalline lens before and after stray light correction. O-ODx in pseudophakic eyes, -ODx in eyes with crystalline lens before correction, - -ODx in eyes with crystalline lens after correction, and -ODx in pseudophakic eyes with crystalline lens in the other eye.

eight-bit black and white camera for ODx measurements, this fundus camera has a 5-megapixel color CCD chip for taking color fundus images.

Nineteen eyes of 19 AMD patients were included in this comparison (group D). These measurements were done on unselected patients in the outpatient department of the University Eye Clinic in Bern, Switzerland. The age of 19 subjects in the group ranged from 60 to 87 years. These individuals were suffering partly from cataracts or had IOL implants.

Optical density was measured sequentially with both methods on the same subjects. Before measurements were made, the subject's pupil was dilated with one drop of mydriaticum (tropicamid $0.5 \%$ and phenylephrin $2.5 \%$ ). As the mean ODx at an annulus with a radius of $0.5 \mathrm{deg}$ is calculated in the standard evaluation in the two-wavelength autofluorescence method, equivalent values were calculated from the results of the one-wavelength reflection method.

\subsection{Comparison of ODx in Eyes of Healthy Subjects and $A M D$ Patients}

The aim of these investigations was to find out any dependence of ODx on age and of differences in ODx between healthy subjects and AMD patients. In measurements in pseudophakic eyes of 22 subjects (group C) with healthy fundus and 45 patients suf-
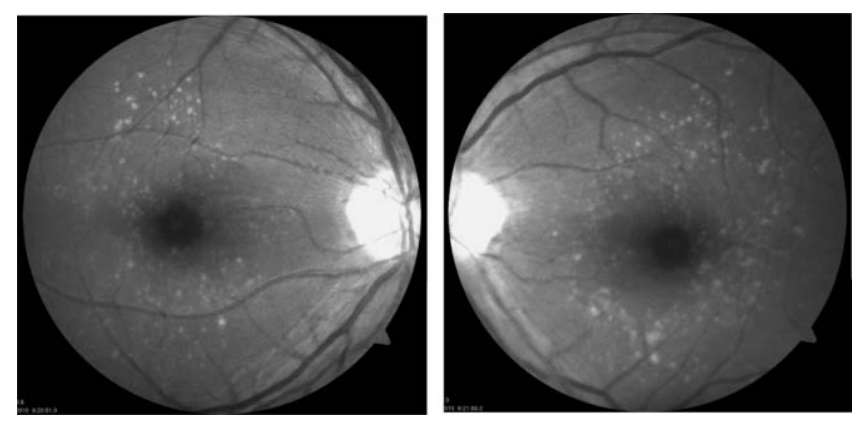

Fig. 5 Blue-light reflection images of a subject, wearing intraocular lenses in both eyes. fering from dry AMD (group E), no stray-light correction was performed because no stray light originates from artificial intraocular lenses. Applying the stray-light correction, ODx was compared in eyes with crystalline lens of 45 healthy subjects (group B) and 125 AMD patients (group F). The program SPSS 15 (IBM Corporation, Armonk, New York) was used for statistical calculations. After the test for Gaussian distribution and for equity of distribution, the unpaired t-test or the Wilcoxon test was used for comparison of ODx in healthy or AMD eyes.

\section{Results}

\subsection{Correction of Optical Density for Stray Light in the Lens}

The normalized age-dependent stray-light equivalent of 232 subjects is given in Fig. 2. In addition to the stray-light equivalent, the optical density of xanthophyll was determined by the method described above. Thus, the decreasing calculated optical density of xanthophyll versus the scattering equivalent is shown in Fig. 3.

Combining both relationships, the decrease in calculated ODx by stray light in the aging lens can be compensated. According to a large study of intraocular stray light as a function of age, a serious increase in stray light can be seen to develop in eyes of patients older than 45 years. ${ }^{46}$ Consequently, a correction function $\triangle \mathrm{ODx}$ for subjects older than 45 years was calculated. This value was added to the measured optical density of xanthophyll as a function of the age of subjects. Equation (3) gives $\triangle \mathrm{ODx}$ as a function of the subject's age $A$.

$$
\begin{aligned}
\Delta \mathrm{ODx}= & \left(-3.5 \times 10^{-9}\right) A^{4}+\left(2.1812 \times 10^{-6}\right) A^{3} \\
& -\left(5.03 \times 10^{-4}\right) A^{2}+0.05085 A-1.455 .
\end{aligned}
$$

The factor $k$ in Eq. (2) was calculated as $k=1.2$, taking into account the extinction of xanthophyll at the wavelength of the blue light $(480 \pm 5 \mathrm{~nm})$ used in relation to the maximal extinction at $460 \mathrm{~nm}^{40}$

\subsection{Reproducibility}

The reproducibility was determined in 10 xanthophyll measurements in the same eye of three subjects in the age of 47,61 , and 78 years. The fundus camera was completely adjusted anew to the subject's eye before each measurement was made. The reproducibility was determined as the coefficient of variation, before and after correction of stray light in the crystalline lens Table 1.

The reproducibility of all parameters is better than $\sim 6 \%$ before stray-light correction. These very good results were reached because the used fundus camera has a focusing tool. After correction for stray light in the lens (maxODx corr), the coefficient of variation is much lower because an age-dependent constant was added to max ODx.

\subsection{ODx in Pseudophakic Eyes and Comparison to Eyes with Crystalline Lens of the Same Subject}

No age dependence of ODx was found for pseudophakic eyes (group C, Fig. 4). In 11 subjects with an artificial IOL in one eye and crystalline lens in the other eye (subgroup of C), the effect of stray-light compensation was demonstrated. After stray-light 
Schweitzer et al.: Simple and objective method for routine detection of the macular pigment xanthophyll

Table 1 Reproducibility of xantophyll measurements in three subjects of different ages.

\begin{tabular}{|c|c|c|c|c|c|c|}
\hline Age & & Volume & Area & mean $O D$ & $\max O D$ & corr. $\max O D$ \\
\hline & & in ODU* $\operatorname{grd}^{2}$ & in $\mathrm{grd}^{2}$ & in ODU & in ODU & in ODU \\
\hline \multirow[t]{3}{*}{47} & Mean & 1,781 & 7,944 & 0,224 & 0,577 & 0,610 \\
\hline & Stand. Dev. & 0,101 & 0,343 & 0,006 & 0,017 & 0,017 \\
\hline & Coeff. Variat. & 0,057 & 0,043 & 0,028 & 0,030 & 0,028 \\
\hline \multirow[t]{3}{*}{61} & Mean & 1,089 & 6,090 & 0,179 & 0,495 & 0,717 \\
\hline & Stand. Dev. & 0,066 & 0,368 & 0,004 & 0,011 & 0,011 \\
\hline & Coeff. Variat. & 0,060 & 0,060 & 0,025 & 0,022 & 0,015 \\
\hline \multirow[t]{3}{*}{78} & Mean & 0,426 & 4,558 & 0,094 & 0,230 & 0,592 \\
\hline & Stand. Dev. & 0,024 & 0,245 & 0,004 & 0,014 & 0,014 \\
\hline & Coeff. Variat. & 0,055 & 0,054 & 0,046 & 0,063 & 0,024 \\
\hline
\end{tabular}

compensation in eyes with crystalline lens, identical ODx were determined in 8 of 11 eyes.

In addition, ODx was determined in the left and right eye of the same subject, wearing IOLs in both eyes. Dark-appearing vessels as well as bright exudates in pixelwise shifted fields of $11 \times 11$ pixels result in an increased coefficient of variation and are not used as nodes for calculating the shading function. Figure 5 shows the corresponding blue-light reflection images of both eyes. As demonstrated in Table 2, nearly identical values were determined for all calculated parameters in both eyes before stray-light correction. The differences between both eyes are below the margins of error of single measurements according to Table 1.

\subsection{Comparison to the Two-wavelength Autofluorescence Method}

The correlation between the one-wavelength reflection method and two-wavelength autofluorescence method is shown in Fig. 6. In this comparison, the optical density of xanthophyll of 19 elderly subjects (group D) was measured using both methods. The age of these AMD patients ranged from 60 to 87 years, and they were suffering from cataract or had IOL implants. No correction for stray light was applied in pseudophakic eyes. A good correlation $\left(R^{2}=0.855\right)$ was achieved between both methods.

Table 2 Comparison of ODx in a subject, wearing intraocular lenses in both eyes.

\begin{tabular}{lcccc}
\hline Eye & mean ODx & max ODx & Volume & Area \\
\hline Right & 0.259 & 0.618 & 2.482 & 9.554 \\
Left & 0.258 & 0.626 & 2.537 & 9.844 \\
\hline
\end{tabular}

\subsection{Optical Density of Macular Pigment in Healthy Eyes and in Dry AMD}

\subsubsection{Comparison in pseudophakic eyes}

The optical density of xanthophyll in pseudophakic eyes of 22 healthy subjects (group C) and 45 eyes of patients suffering from dry AMD (group E) is shown in Fig. 7. No correction for stray light was applied in these measurements. There was no significant dependence of ODx on age in both groups. But ODx in healthy subjects $(0.615 \pm 0.103$ ODU) was significantly higher than in AMD $(0.491 \pm 0.102$ ODU $),(p=0.00003$, Fig. 8).

\subsubsection{Comparison in eyes with crystalline lens}

In 45 measurements (group B) of ODx in healthy subjects and 125 dry AMD patients (group F), the correction for stray light

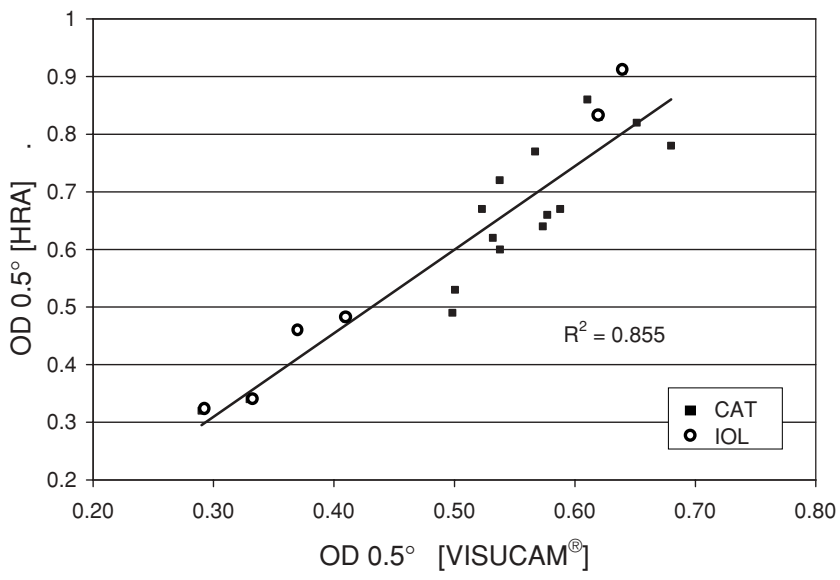

Fig. 6 Correlation between xanthophyll measurements using the one wavelength reflection method and the two wavelengths autofluorescence method. Ages of these dry AMD patients ranged from 60 to 87 years. 


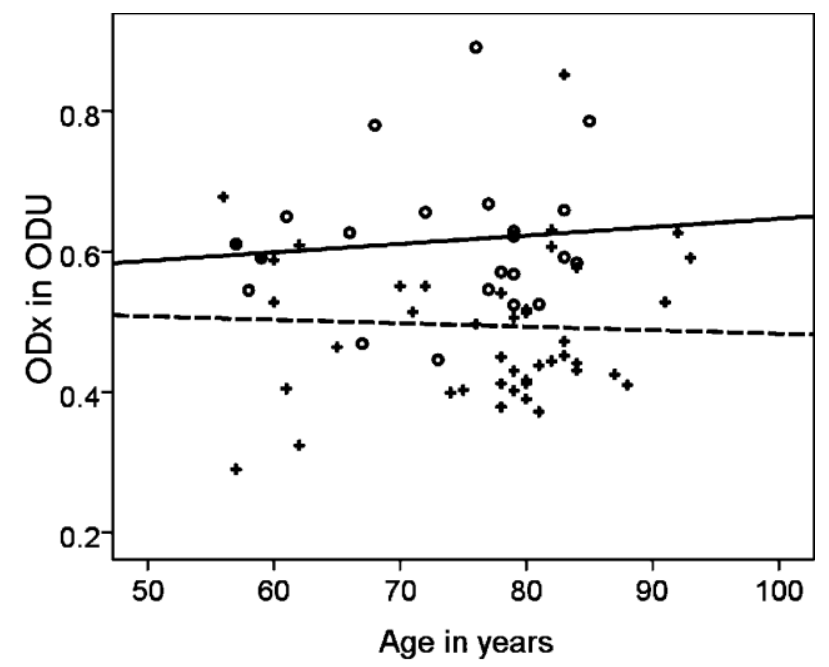

Fig. 7 ODx in pseudophakic eyes of 22 healthy subjects and 45 AMD patients: $\mathbf{O}$, - healthy subjects $\left(\mathrm{ODx}=0.0012 \mathrm{Age}+0.5271, R^{2}\right.$ $=0.011)$ and,+---- AMD patients $(\mathrm{ODx}=-0.0003 \mathrm{Age}+0.533$, $\left.R^{2}=0.001\right)$.

was applied. There was also no dependence on age for ODx for both groups (Fig. 9). As also measured in pseudophakic eyes, ODx in healthy subjects $(0.674 \pm 0.098$ ODU) was higher than in $\operatorname{AMD}(0.627 \pm 0.093$ ODU) ( $p=0.00012$, Fig. 10).

Despite the high variation of ODx of about a factor of 3 in the AMD group, the difference between the means in both groups was highly significant.

\section{Discussion}

A high optical density of the macular pigment xanthophyll is assumed to be a factor offering protection against the development of age-related macular degeneration. Several highly sophisticated methods have been described for use in the subjective and objective measurement of the optical density of xanthophyll in the retina. ${ }^{27,29-37}$

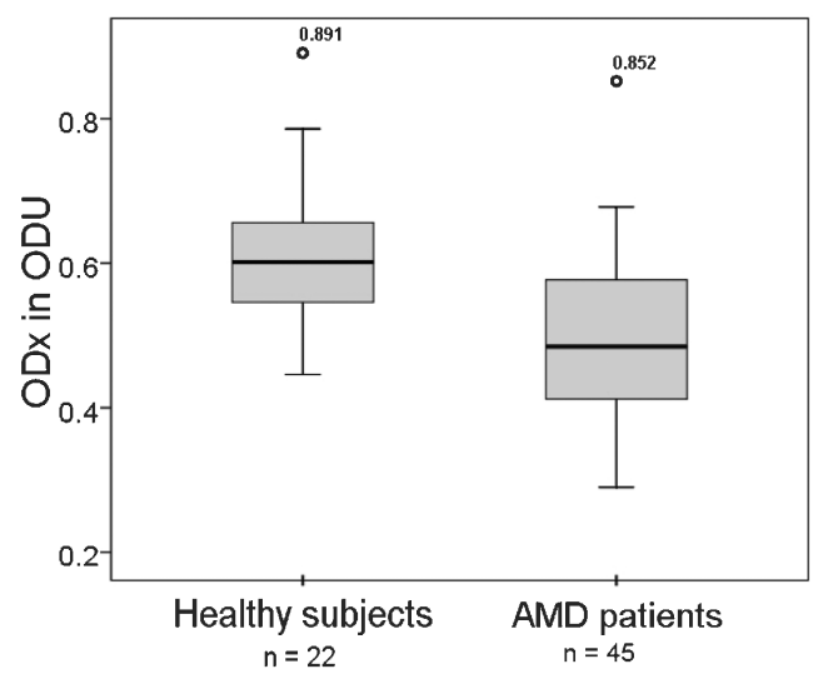

Fig. 8 Boxplot of ODx in pseudophakic eyes of subjects with healthy fundus $(0.615 \pm 0.103$ ODU) and of AMD patients $(0.491 \pm 0.102$ ODU). The difference is highly significant $(p=0.00003)$.

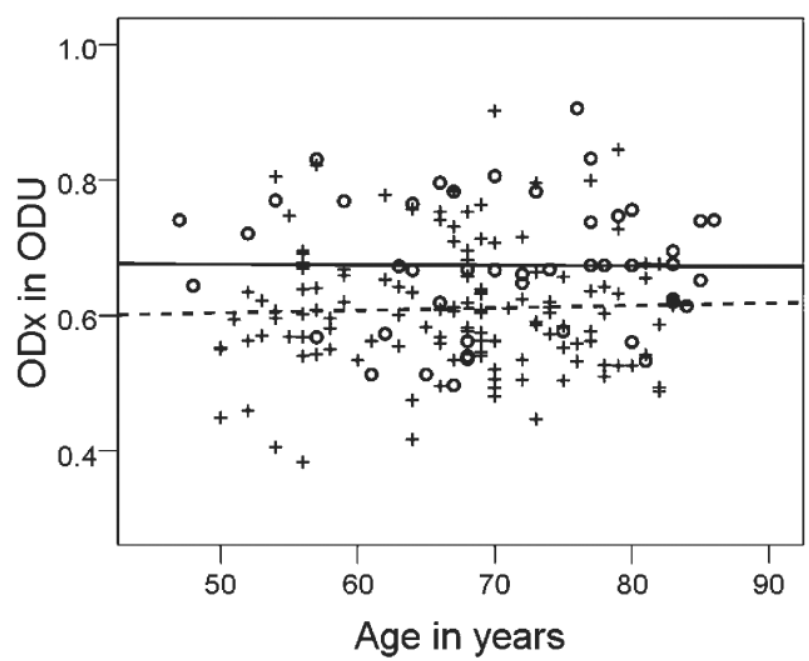

Fig. $9 \mathrm{ODx}$ in eyes with crystalline lens in 45 healthy subjects and in 125 dry AMD patients: $\mathbf{O}$-healthy subjects (ODx = $-2 \times 10^{-6}$. Age $+0.6744, R^{2}=5 \times 10^{-8}$ ) and + -AMD patients $\left(\mathrm{ODx}=4 \times 10^{-4}\right.$. Age $\left.+0.580, R^{2}=1.2 \times 10^{-3}\right)$.

On the basis of our own experience, simple, objective, and quick methods are needed for a wide application of xanthophyll measurements in clinical practice. The reduction from two wavelengths to one wavelength in fluorescence measurements of xanthophyll is a possibility. ${ }^{45}$ Unfortunately, the advantage of the two-wavelength method of being independent of nonuniform fundus illumination is lacking in the one-wavelength method. In this method, the fluorescence light depends on the intensity of the excitatory light.

The method, described here, can be applied in commonly used fundus cameras or in laser scanner ophthalmoscopes. In principle, the same technique can be used as in fluorescence angiography. The fundus is illuminated by blue light, preferably near the absorption maximum of xanthophyll at $460 \mathrm{~nm}$. For detection of a blue-light reflection image, the fluorescenceblocking filter must be removed. The individual fundus

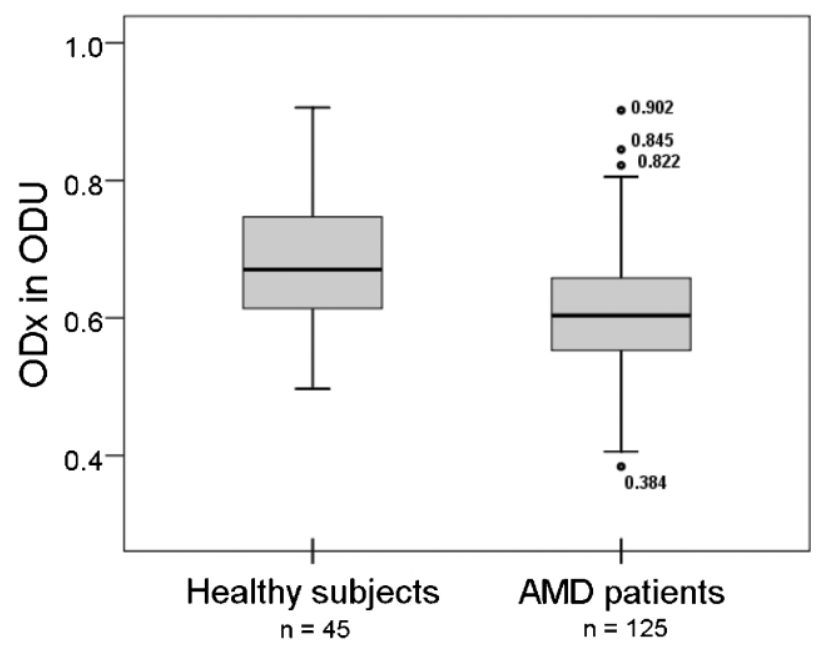

Fig. 10 Boxplot of ODx in eyes with crystalline lens of 45 healthy subjects: (ODx $=0.674 \pm 0.098$ ODU) and of 125 AMD patients (ODx $=0.627 \pm 0.093$ ODU), $p=0.00012$. 


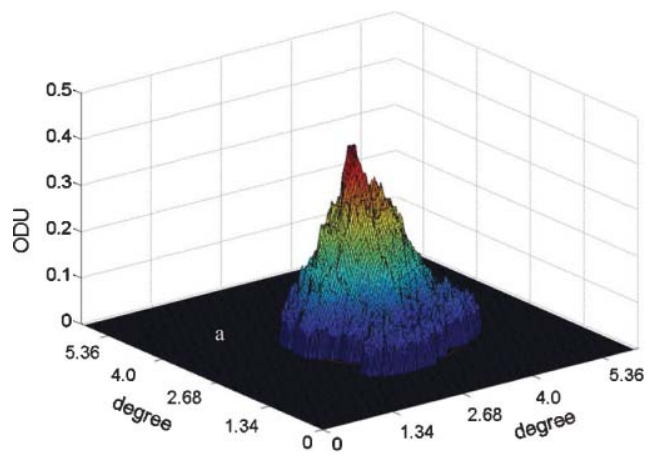

(a)

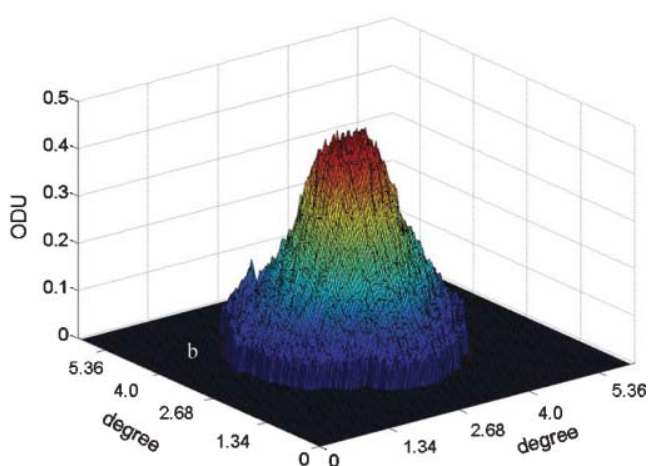

(b)

Fig. 11 Comparison of parameters of ODx measurement using one-wavelength reflection method of two patients. Subject (a): volume $=0.826$ ODU $\bullet \operatorname{grd}^{2}$, area $=6.015 \mathrm{grd}^{2}$, mean ODx $=0.137 \mathrm{ODU}, \max$ ODx $=0.422 \mathrm{ODU}$, subject (b): volume $=1.786 \mathrm{ODU}_{\bullet} \operatorname{grd}^{2}, \operatorname{area}=0.742 \mathrm{grd}^{2}$, mean ODx $=0.166$ ODU, and $\max$ ODx $=0.468$ ODU.

reflection, as well as inhomogeneities of the illumination, is calculated automatically as a shading function. Nodes for the construction of the shading function are determined from outside the macula. Inside the macula, the shading function is used as a virtual background reference for the calculation of the optical density of xanthophyll. Thus, nonhomogeneities in fundus reflectance are not considered.

As stray light in the crystalline lens acts as an additive contribution, both in the numerator and the denominator, a severe cataract has a diminishing effect on the calculated optical density of xanthophyll. This effect is compensated by a correction term. This term was determined from normalized stray-light measurements versus age in subjects older than 45 years (group A) and ODx versus stray-light equivalent (group B). Starting from this age, increased stray light in the lens is described in the literature. ${ }^{46}$

In contrast to crystalline lens, no stray light will originate form artificial intraocular lenses. Agreeing with this assumption, no dependence on age was found for ODx in 22 pseudophakic eyes (group C). This is in agreement with a study in which changes in the optical density of xanthophyll with age were measured using different methods. ${ }^{47}$ A weak decrease in optical density of macular pigment on age was found by heterochromatic flicker photometry. ${ }^{48}$ The corresponding $R^{2}=0.082$ was very small.

The effect of stray-light compensation was demonstrated by comparing the optical density of xanthophyll in 11 subjects with crystalline lens in one eye and an artificial IOL in the other eye (subgroup of C). In this group, the values of ODx were equivalent in both eyes after stray-light compensation. Only for two subjects, aged 61 and 67 years, there is a residual difference in ODx between both eyes. The stray light in the crystalline lens of both subjects was probably higher than in lenses of group B, used for the determination of lens scattering. Differences in ODx between both eyes of the same subject cannot be excluded. ${ }^{49}$ No stray-light correction is applied in pseudophagic eyes. Measurements in both IOL-wearing eyes of the same subject result in nearly the same values for max ODx, mean ODx, area, and volume.

The stray-light compensation can be improved by individual stray-light measurements. In this case, the fundus would not be completely illuminated. Then, the stray light is the light measured from a nonilluminated fundus region. This solution requires changes in the illumination system (e.g., correction of myopic or hyperopic errors), which can be done by producers of ophthalmologic devices only, not by residential doctors.

Despite the fact that the one-wavelength reflectance method is a simplified one, the correlation is excellent with the results obtained with the highly sophisticated two-wavelength autofluorescence method for the determination of the optical density of xanthophyll.

In addition to heterochromatic flicker photometry, twowavelength reflection or autofluorescence method, not only the maximum ODx, but also the mean ODx, the area of extension of xanthophyll, and the "volume" of xanthophyll were calculated by the one wavelength reflection method described here. The volume of the macular pigment, especially, seems to be important. This parameter takes into account differences in the local distribution of xanthophyll. The magnitude of the volume is equivalent to the number of molecules of lutein and zeaxanthin. Therefore, it can be considered to be a measure of the protective capacity of the macular pigment. Despite comparable maximal ODx, the volume can be considerably different. As an example, the quasi three-dimensional distribution of ODx is shown in Fig. 11. Also in the case of the comparable maximum ODx (subject a: $\max$ ODx $=0.422$ ODU, subject $\mathrm{b}$ : $\max$ ODx $=0.468$ ODU), the volume in subject $\mathrm{b}$ is twice the volume in subject a. Furthermore, the distribution of xanthophyll is not symmetric in subject a.

The reproducibility of the parameters, with a coefficient of variation of $<6 \%$ before stray-light correction, is sufficient for the individual determination of macular pigment and its increase following supplementation, for example, lutein. In addition to measurements of xanthophyll in healthy subjects, the one-wavelength reflection method was also applied to patients suffering from dry AMD. Here, no dependence of xanthophyll on age was found for AMD patients both in 45 pseudophakic eyes (group E) and in 125 eyes with crystalline lens (group F). In the statistical comparison between healthy subjects and AMD patients, ODx was significantly lower in dry AMD than in healthy subjects, both in pseudophakic eyes and in eyes with crystalline lens. Thus, the assumption can be confirmed that the reduced optical density of xanthophyll might be a risk factor of AMD. As the correction for stray light in the crystalline lens was determined as normative mean, the age 
distribution should be identical in groups of healthy subjects and AMD patients.

A weak decrease in the virtual background reflection caused by an increased optical density of melanin in the fovea will be compensated by the calculated shading function. If the increase in the optical density of melanin is steep in the fovea, then the calculated ODx might be too high.

The presented method is a simplification of the radiation transport at the ocular fundus. The globally determined parameters for characterization of the macular pigment xanthophyll proved helpful in clinical practice, especially for evaluating the effect of lutein supplementation. To determine influences or alterations of optical properties in the single fundus layers, e.g., in developing of age-related macular degeneration, proper physical models are necessary.

\section{Conclusions}

The one-wavelength reflection method was shown to be a simple, objective method for xanthophyll measurements in clinical routine. Besides in clinical studies, this method can be used by practitioners. The applied compensation for stray light in an elderly crystalline lens results in comparable optical densities of xanthophyll in both the pseudophakic eye and in the eye with crystalline lens of the same subject. There was a good correlation of the one-wavelength reflection method to the two-wavelength autofluorescence method in xanthophyll measurements of AMD patients both with pseudophakic eyes and eyes with a crystalline lens. Furthermore, the optical density of xanthophyll did not depend on age in pseudophakic eyes both in dry AMD and in eyes with healthy fundus.

Despite the high individual variability, the optical density of macular pigment was significantly lower in dry AMD than in age-matched healthy subjects. Thus, a supplementation by lutein and zeaxanthin might be helpful in endangered subjects e.g. with AMD in the family history.

\section{References}

1. C. C. Klaver, R. C. Wolfs, J. R. Vingerling, A. Hofmann, and P. T. de Jong, "Age-specific prevalence and causes of blindness and visual impairment in an older population: the Rotterdam study," Arch. Ophthalmol. 116(5), 653-658 (1998).

2. P. Bhosale, B. Serban, and P. S. Bernstein, "Retinal carotenoids can attenuate formation of A2E in retinal pigment epithelium," Arch. Biochem. Biophys. 483, 175-181 (2009).

3. C. A. Parish, M. Hashimoto, K. Nakanishi, J. Dillon, and J. R. Sparrow, "Isolation and one step preparation of A2E and iso-A2E, fluorophoresfrom human retinal pigment epithelium," Proc. Natl. Acad. Sci. U S A 95, 14609-14613 (1998).

4. G. E. Eldred, "Lipofuscin fluorophore inhibits lysosomal protein degradation and may cause early stages of macular degeneration," Gerontology 41 (Suppl. 2), 15-28 (1995).

5. S. P. Sundelin and S. E. G. Nilsson, "Lipofuscin-formation in retinal pigment epithelial cells is reduced by antioxidants," Free Radic. Biol. Med. 31(2), 217-225 (2001).

6. M. A. Zarbin, "Current concepts in pathogenesis of age-related macular degeneration," Arch. Ophthalmol. 122, 598-614 (2004).

7. M. Rozanovska, J. Jarvis-Evans, W. Korytkowski, M. E. Boulton, J. M. Burke, and T. Sarna, "Blue light-induced reactivity of retinal age pigment: in vivo generation of oxygen reactive species," J. Biol. Chem. 270, 18825-18830 (1995).

8. M. Rozanovska, J. Wessel, M. E. Boulton, J. M. Burke, M. A. Rodgers, T. G. Truscott, and T. Sarna, "Blue light-induced singlet oxygen gener- ation by retinal lipofuscin in non-polar media," Free Radic. Biol. Med. 24, 1107-1112 (1998).

9. U. Wihlmark, A. Wrigstad, K. Roberg, S. E. G. Nilsson, and U. T. Brunk, "Lipofuscin accumulation in cultured retinal pigment epithelial cells causes enhanced sensitivity to blue light irradiation,"Free Radic. Biol. Med. 22, 1229-1234 (1997).

10. C. S. Foote, C. Y. Chang, and R. W. Denny, "Chemistry of singlet oxygen. X. carotenoid quenching parallels biological protection," $J$. Am. Chem. Soc. 92, 5216-5218 (1970).

11. K. Kirchfeld, "Carotenoid pigments: their role in protecting against photooxidation in eyes and photoreceptor cells," Proc. R. Soc. Lond. 216, 71-85 (1982)

12. D. M. Snodderly, "Evidence for protection against age related macular degeneration by carotenoids and antioxidant vitamins," Am. J. Clin. Nutr. 62 (Suppl.), 1448-1460 (1995).

13. B. R. Wooten and B. R. Hammond, "Spectral absorbance and special distribution of macular pigment using hererochromic flicker photometry," Optom. Vis. Sci. 82, 378-386 (2005).

14. E. L. Loane, J. M. Nolan, O. O'Donovan, P. Bhosale, P. S. Bernstein, and S. Beatty, "Transport and retinal rapture of lutein and zaxanthin with reference to age-related macular degeneration," Surv. Ophthalmol. 53(1), 68-81 (2008).

15. R. A. Bone, J. T. Landrum, and S. L. Tarsis, "Preliminary identification of the human macular pigment," Vis. Res. 25, 1531-1539 (1985).

16. J. T. Landrum and R. A. Bone, "Minireview lutein, zeaxanthin, and the macular pigment," Arch. Biochem. Biophys. 385(1), 28-40 (2001).

17. M. D. Snodderly, J. D. Auran, and F. C. Delori, "The macular pigment. II: Spatial distribution in primate retinas," Invest. Ophthalmol. Vis. Sci. 25, 674-685 (1984).

18. E. W. Chong, T. Y. Wong, A. J. Kreis, J. A. Simpson, and R. H. Guymer, "Dietary antioxidants and primary prevention of age related macular degeneration: systematic review and meta-analysis,"British Medical Journal 335(7623), 755-759 (2007).

19. J. P. SanGiovanni, E. Y. Chew, T. E. Clemons, F. L. Ferris 3rd, G. Gensler, A. S. Lindblad, R. C. Milton, J. M. Seddon, R. D. Sperduto, and Age-Related Eye Disease Study Research Group, "The relationship of dietary carotenoid and vitamin A, E, and C intake with age-related macular degeneration in a case-control study: AREDS Report No. 22," Arch. Ophthalmol. 125(9), 1225-1232 (2007).

20. S. Richer, W. Stiles, L. Statkute, J. Pulido, J. Frankowski, D. Rudy, K. Pei, M. Tsipursky, and J. Nyland, "Double-masked, placebo-controlled, randomized trial of lutein and antioxidant supplementation in the intervention of atrophic age-related macular degeneration: the Veterans LAST study (lutein antioxidant supplementation trial),"Optometry 75(4), 216-230 (2004).

21. V. M. Flood, W. Smith, E. Rochtchina, J. J. Wang, and P. Mitchell, "Assembling a nutrient database for a large cohort study: the Blue Mountains Eye Study," Food Austral. 60(1-2) 37-41 (2008).

22. E. Cho, J. M. Seddon, B. Rosner, W. C. Willett, and S. E. Hankinson, "Prospective study of intake of fruits, vegetables, vitamins, and carotenoids and risk of age-related maculopathy," Arch Ophthalmol. 122(6), 883-892 (2004).

23. G. M. Van Den Langenberg, J. A. Mares-Perlman, R. Klein, B. E. K. Klein, W. E. Brady, and M. Palata, "Associations between antioxidant and zinc intake and the 5-year incidence of early age-related maculopathy in the Beaver Dam Eye Study," Am. J. Epidemiol. 148, 204-214 (1998).

24. E. W. Chong, A. J. Sinclair, and R. H. Guymer, "Facts on fats," Clin. Exp. Ophthalmol. 34(5), 464-471 (2006).

25. R. van Leeuwen, S. Boekhoorn, J. R. Vingerling, J. C. Witteman, C. C. Klaver, A. Hofman, and P. T. de Jong, "Dietary intake of antioxidants and risk of age-related macular degeneration," J. Am. Med. Assn. 294(24), 3101-3107 (2005).

26. S. M. Moeller, N. Parekh, L. Tinker, C. Ritenbaugh, B. Blodi, R. B. Wallace, J. A. Mares, and "CAREDS Research Study Group, "Associations between intermediate age-related macular degeneration and lutein and zeaxanthin in the Carotenoids in Age-related Eye Disease Study (CAREDS): ancillary study of the Women's Health Initiative," Arch. Ophthalmol. 124(8), 1151-1162 (2006).

27. R. A. Bone, J. T. Landrum, and A. Cains, "Optical density spectra of the macular pigment in vivo and in vitro," Vis. Res. 32(1), 105-110 (1992). 
28. J. T. Landrum, R. A. Bone, H. Joa, M. D. Kilburn, L. L. Moore, and K. E. Sprague, "A one year study of the macular pigment: the effect of the 140 days of lutein supplementation," Exp. Eye Res. 65, 57-62 (1997).

29. B. R. Hammond, E. J. Johnson, R. M. Russell, N. Krinsky, K. J. Yeum, R. B. Edwards, and M. D. Snodderly, "Dietary modification of human macular pigment density," Invest. Ophthalmol. Vis. Sci. 38, 1795-1801 (1997).

30. A. E. Elsner, S. A. Burns, F. C. Delori, and R. H. Webb, "Quantitative reflectometry with the SLO," Chapter 10 in Laser Scanning Ophthalmoscopy and Tomography, J. E. Nasemann and R. O. W. Burk Eds., pp. 109-121, Quintessenz, Muenchen (1990).

31. D. van Norren and L. F. Tiemeijer, "Spectral reflectance of the human eye,"Vis. Res. 26(2), 313-320 (1986).

32. T. T. Berendschot, R. A. Goldbohm, W. A. Klopping, J. van de Kraats, J. van Norel, and D. van Norren, "Influence of lutein supplementation on macular pigment, assessed with two objective techniques," Invest. Ophthalmol. Vis. Sci. 41(11), 3322-3326 (2000).

33. J. van de Kraats, M. J. Kanis, S. W. Genders, and D. van Norren, "Lutein and zeaxanthin measured separately in the living human retina with fundus reflectometry," Invest. Ophthalmol. Vis. Sci. 49(12), 55685573. (2008).

34. N. P. A. Zagers and D. van Norren, "Absorption of the eye lens and macular pigment derived from the reflectance of cone photoreceptors," J. Opt. Soc. Am. A 21(12), 2257-2268 (2004).

35. D. Schweitzer, M. Hammer, and M. Scibor, "Imaging spectrometry in ophthalmology-principle and applications in microcirculation and in investigation of pigments," Ophthalmic. Res. 28(Suppl 2), 37-44 (1996).

36. F. C. Delori, D. G. Goger, B. R. Hammond, M. D. Snodderly, and S. A. Burns, "Macular pigment density measured by autofluorescence spectrometry: comparison with reflectometry and heterochromatic flicker photometry," J. Opt. Soc. Am. A 18(6), 1212-1230 (2001).

37. P. S. Bernstein, M. D. Yoshida, N. B. Katz, R. W. Mc Clane, and W. Gellermann, "Raman detection of macular carotenoid pigments in intact human retina," Invest. Ophthalmol. Vis. Sci. 39(11), 2003-2011 (1998).

38. D. Schweitzer, "In Vivo-Detection of macular pigment and its effect by lutein supplementation." Chapter 12 in Focus on Macular Degeneration
Research, O. R. Ioseliani, Ed., pp. 253-257, Nova Science, New York (2003).

39. S. Beatty, F. J. G. M. van Kuijk, and U. Chakravarthy, "Macular pigment and age-related macular degeneration: longitudinal data and better techniques of measurement are needed," Invest. Ophthalmol. Vis. Sci. 49(3), 843-845 (2008)

40. G. Wyszecki and W. S. Stiles, Colour Science: Concepts and Methods, Quantitative Data and Formulae, 2nd ed. Wiley, Hoboken, NJ (1982).

41. R. T. Smith, T. Nagasaki, J. R. Sparrow, I. Barbazetto, C. C. W. Klaver, and J. K. Chan, " A method of drusen measurement based on the geometry of fundus reflectance," Biomed. Eng. Online 210 April 18 (2003).

42. L. Leistritz and D. Schweitzer, "Automated detection and quantification of exudates in retinal images," Proc. SPIE 2298, 690-696 (1994).

43. H. Wustemeyer, C. Jahn, A. Nestler, T. Barth, and S. Wolf, "A new instrument for the quantification of macular pigment density: first results in patients with AMD and healthy subjects," Graefes. Arch. Clin Exp. Ophthalmol. 240, 666-671 (2002).

44. M. Jarc-Vidmar, A. Kraut, and M. Hawlina, "Fundus autofluorescence imaging in Best's vitelliform dystrophy," Klin, Monbl. Augenheilkd. 220(12), 861-867 (2003)

45. M. Trieschmann, B. Heimes, H. W. Hense, and D. Pauleikhoff, "Macular pigment optical density measurement in autofluorescence imaging: comparison of one- and two-wavelength methods," Graefes. Arch. Clin Exp. Ophthalmol. 244(12), 1565-1574 (2006).

46. T. J. T. P. van den Berg, L. J. van Rijn, R. Michael, C. Heine, T. Coeckelbergh, C. Nischler, H. Wilhelm, G. Grabner, M. Emesz, R. I. Barraquer, J. E. Coppens, and L. Franssen, "Straylight effects with aging and lens extraction," Am. J. Ophthalmol. 144, 358-363 (2007).

47. T. T. J. M. Berendschot and D. van Norren, "On the age dependency of the macular pigment optical density," Exp. Eye Res. 81(5), 602-609 (2005).

48. J. M. Loan, J. Stack, O. O’Donavan, E. Loan, and S. Betty, "Risk factors for age-related maculopathy are associated with a lack of macular pigment," Exp. Eye Res. 84, 61-74 (2007).

49. M. J. Kanis, T. T. J. M. Berendschot, and D. van Norren, "Interocular agreement in melanin and macular pigment optical density," Exp. Eye Res. 84, 934-938 (2007). 